\title{
Mechanical Demands at the Ankle Joint During Saut de Chat and Temps levé Jumps in Classically Trained Ballet Dancers
}

This article was published in the following Dove Press journal: Open Access Journal of Sports Medicine

\author{
Sarah K Perry \\ Harsh H Buddhadev (D) \\ Lorraine R Brilla (D) \\ David N Suprak
}

Department of Health and Human Development, Western Washington University, Bellingham, WA, USA
Correspondence: Harsh $\mathrm{H}$ Buddhadev Department of Health and Human Development, Western Washington University, 20IH Carver Academic Facility, MS 9067, 516 High Street, Bellingham, WA 98225, USA

Tel + I $360650-4 \mid I 5$

Fax +I360650-7447

Email harsh.buddhadev@wwu.edu
Background: During ballet, injuries to the Achilles tendon are associated with the take-off phase of various jumps.

Research question: The purpose of the study was to assess differences in mechanical demand on the body, specifically at the ankle, in two single-leg jumps commonly trained in ballet: a saut de chat (SDC) and a temps levé (TL).

Methods: Fifteen classically trained female dancers had 16 reflective markers placed on the lower body and each dancer performed each jump three times on a force plate. The marker position data and ground reaction forces (GRF) were captured synchronously at $250 \mathrm{~Hz}$ and $1000 \mathrm{~Hz}$, respectively. Peak vertical GRF, mean rate of force development (RFD), peak ankle moment, and peak ankle power were determined and averaged across trials. Paired $t$-tests were used to determine differences between the SDC and the TL.

Results: When compared to the TL, the SDC displayed significantly higher peak vertical GRF $(p=0.003)$, RFD $(p=0.002)$, and peak ankle moment and power $(p<0.001)$. The effect sizes for these differences were large for all variables (Cohen's $d>0.80$ ).

Conclusion: The mechanical demand at the ankle joint is significantly greater for the SDC than the TL.

Keywords: achilles tendinopathy, power, moment, rate of force development

\section{Introduction}

Ballet is a unique form of dance that requires a high level of athleticism while maintaining a specific visual aesthetic. ${ }^{1,2}$ The artistic requirements of classical ballet dictate physical training and performance. ${ }^{2}$ Unlike other forms of physical activity, the performance of athletic acts such as jumps in ballet primarily serves to achieve the desired aesthetic rather than maximal performance. One of the most athletic aspects of ballet can be observed during jumping movements. ${ }^{3}$ These movements place a high mechanical demand for rapid muscular effort on the lower extremity and are associated with injuries at the ankle joint. ${ }^{4-12}$ Due to the prevalence of impact injuries, ${ }^{4,8}$ a majority of previous research in the discipline of ballet has focused on the landing phase of the jump. ${ }^{13-16}$ However, common injuries, such as Achilles tendinopathy and Achilles tendon (AT) rupture, are associated with the take-off phase of the jump. ${ }^{10,11}$ While altered kinematics have been observed during the take-off phase of jumps in dancers with Achilles tendinopathy, ${ }^{12}$ no research has examined the kinetic demands at the ankle joint during the take-off phase of different ballet jumps in healthy dancers. 
Many jumps specific to ballet involve rapid take-off from a single leg and place a high mechanical demand on the tendons and muscles around the ankle joint. Two of the most frequently performed jump types that have similar take-off strategies but differ in the landing technique are a jeté and a temps levé. ${ }^{3}$ A jeté is a jump that takes off from a single foot and lands on the other. The saut de chat (SDC) is a specific jeté in which a dancer takes off, jumps with high vertical and horizontal propulsive effort, performs the splits mid-air, and lands on the opposite foot. ${ }^{17}$ A temps levé (TL) is a jump type in which the dancer takes off and lands on the same foot. A common type of temps levé jump requires the dancers to take a single step forward and then take-off with propulsion mainly in the vertical direction. $^{3}$

From a mechanical perspective, we speculate singleleg jumps such as SDC which involve a combined vertical and horizontal propulsion would place substantially greater propulsive demands on the lower extremity, specifically the ankle joint, compared to jumps such as TL that primarily involve vertical direction propulsion. The mechanical demand on the whole body during jumping movements can be determined by examining peak vertical ground reaction forces (GRF) and the rate of force development (RFD). ${ }^{18}$ Similarly, peak net joint moments and powers can be used to quantify the mechanical demand about the ankle specifically. ${ }^{19-23}$ To date, no research has examined the differences in mechanical demand at the ankle joint during the take-off phase of different ballet jumps. Determining these differences may provide insights for training progression, ${ }^{17,24}$ injury risk, ${ }^{6-10,12}$ and rehabilitation for dancers with Achilles tendinopathy. ${ }^{12,25}$

The purpose of this study, therefore, was to examine differences in the mechanical demand on the ankle joint in classically trained ballet dancers during two common dance jumps (SDC and TL) that take-off from a single foot. We hypothesized that the mechanical demand on the ankle joint would be greater for the SDC compared to the TL.

\section{Materials and Methods}

\section{Participants}

Fifteen female classically trained ballet dancers (age: 20.7 \pm 2.7 years; height: $1.6 \pm 0.1 \mathrm{~m}$; mass: $56.4 \pm 3.98 \mathrm{~kg}$; training: $13.9 \pm 5.0$ years) participated in the study. The inclusion criteria were no current injury, no major prior injury in the past 5 years that required more than a month off from dance training or surgery, no previous Achilles tendinopathy, and more than 3 years of training in classical ballet at the advanced level. ${ }^{26,27}$ All participants signed a written informed consent prior to participation. The University Institutional Review Board approved the study, and it was carried out in accordance with the principles of the Declaration of Helsinki.

\section{Data Collection}

Participants completed a single testing session lasting approximately $1 \mathrm{hr}$. The session began with 10 mins of standardized ballet barre warm-up exercises followed by measurement of anthropometric characteristics such as body mass, height, leg length, inter-anterior superior iliac spine horizontal distance, as well as knee and ankle widths. The knee and ankle widths were measured as horizontal distances between the medial and lateral epicondyles of the femur and the medial and lateral malleoli at the ankle, respectively. Participants wore tight-fitting spandex clothes, and 16 reflective markers were placed bilaterally on participants' feet, legs, and thigh and around the pelvis according to Plug-in-Gait lower body model guidelines (Vicon, Centennial, Colorado, USA). Threedimensional lower extremity marker position data were collected using a 10-camera motion capture system (Vicon, Vantage V8, Centennial, Colorado, USA) sampling at $250 \mathrm{~Hz}^{28,29} \mathrm{GRF}$ and moment data were collected using two in-ground force plates (Advanced Mechanical Technology Inc., Waterton, MA, USA) sampling at 1000 $\mathrm{Hz}^{27}$

Each participant performed the two different jump conditions: SDC and $\mathrm{TL}^{3,17}$ with their preferred take-off leg in a random order. For the SDC, the participants were asked to place their preferred take-off leg forward onto the first force plate. They then jumped, bringing their other leg forward and performing the splits in midair before landing on a second force plate on their other leg (Figure 1). The TL began with an identical approach with the participants placing their preferred take-off leg forward onto the force plate. They then jumped vertically while keeping their other leg behind their take-off leg and landing on the take-off leg (Figure 2). Preceding each jump condition, participants were shown a video of the jump and then they performed three familiarization jumps on the force plate. The participants then performed three successive jumping trials for each jump condition. ${ }^{13}$ Marker position and GRF data were captured synchronously using the Nexus software (Vicon Nexus version 2.6, Centennial, Colorado, USA). 


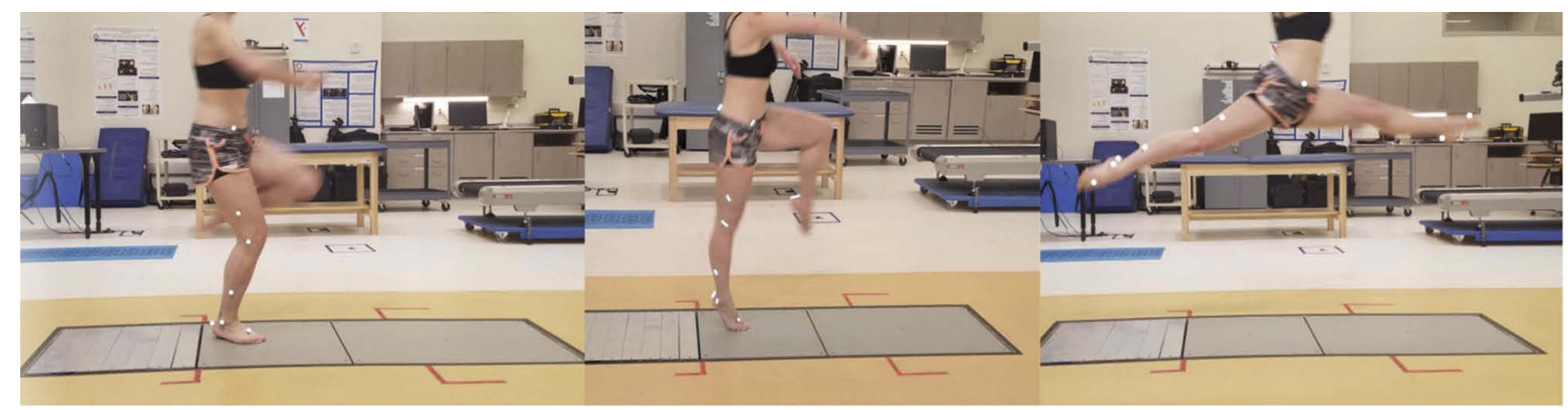

Figure I Experimental set-up for the saut de chat (SDC) jump.

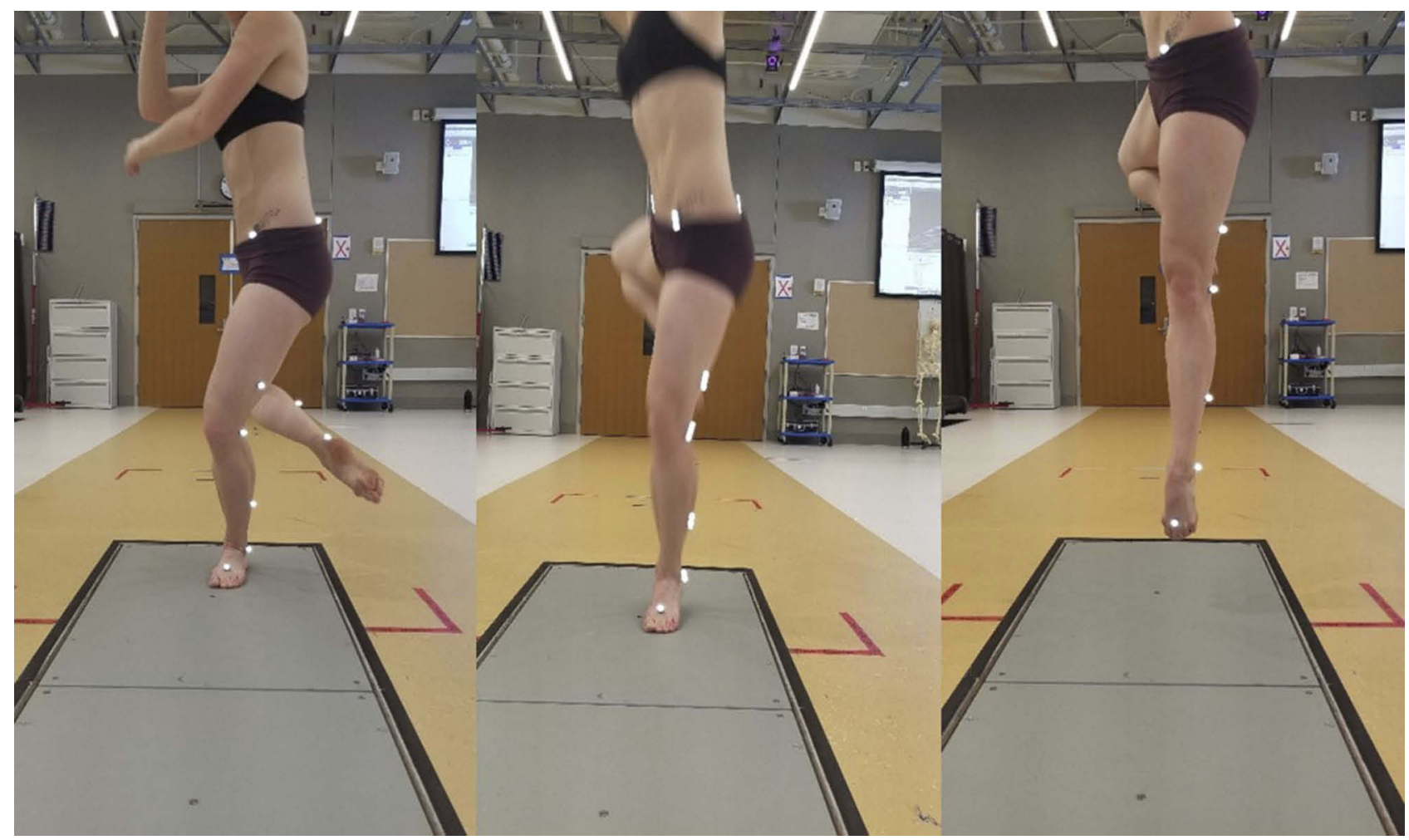

Figure 2 Experimental set-up for the temps levé (TL) jump.

During both jump conditions, the dancers were encouraged to give maximal effort, emphasizing maximal jump height. The jump conditions were separated by a 1-min rest period. ${ }^{30,31}$ SDC and TL with lower body marker placement are shown in Figures 1 and 2, respectively.

\section{Data Analysis}

Flight time for the jump was identified using the force plate data from the instant of take-off to landing, during which time the vertical GRF was zero N. Duration of the take-off phase of the jump was defined from the instant of the dancer stepping on the force plate (i.e., when vertical
GRF exceeded $20 \mathrm{~N})^{32,33}$ to becoming airborne (i.e., when vertical GRF was $0 \mathrm{~N}$ ). All dependent variables were identified during the take-off phase of the jump. The GRF data were normalized to body mass and then the peak values were identified for the vertical component of GRF for each trial. The mean rate of force development (RFD) was computed as the slope of the change in vertical GRF from minimum to peak over the corresponding change in time. Using marker position data, a rigid 7-segment (i.e., pelvis and left and right femur, tibia, and feet segments) linked lower extremity model was created via the Plug-in Gait pipeline. ${ }^{34}$ Markers placed on the lower 
body segments were used to create rotational matrices for calculating cardan segment and joint angles. A flexion/ extension, abduction/adduction, and internal/external rotation sequence was used. Segment masses, centers of mass locations, and moments of inertia were estimated by entering anthropometric measurements into Dempster's equations. $^{35}$ The angular and center of mass velocities and accelerations of each segment were calculated using finite difference equations. An inverse dynamics approach was then used to determine sagittal plane net joint moments and forces at the ankle joint via the dynamic Plug-in Gait pipeline in Nexus software. ${ }^{34}$ For each trial, peak moment and power values were identified for the take-off phase and then normalized to body mass. For all variables, the data were averaged across the three trials for each condition.

\section{Statistical Analysis}

For each variable, paired $t$-tests were used to determine differences between the SDC and TL conditions. The alpha level was set at 0.05 . The effect sizes were calculated as Cohen's $d$. Small, medium, and large effect sizes correspond to Cohen's $d$ values of $0.20,0.50$, and 0.80 , respectively. ${ }^{36}$ All statistical analyses were performed using the SPSS software version 25 (Chicago, IL, USA, 2011).

\section{Results}

For one participant, we were not able to obtain marker position data due to a technical error. Therefore, peak ankle moment and peak ankle power were reported for 14 participants, whereas peak vertical GRF, average RFD, and flight time were reported for 15 participants. All dependent variables were significantly higher during the SDC than during the TL $(p<0.05$; Table 1$)$. The mean difference between conditions for all variables was large (Cohen's $d>0.80$; Table 1), with the difference between peak ankle power showing the largest effect size (Cohen's $d=2.290$ ). Additionally, all dancers showed very similar jump profiles for vertical GRF, ankle moment, and ankle power during the propulsive phase of the jump. Representative data for vertical GRF, ankle moment, and ankle power during take-off are presented in Figures 3-5.

\section{Discussion}

The purpose of this study was to examine differences in demand on the ankle joint during two common dance jumps (SDC and TL). We hypothesized that the SDC would place a greater demand on the ankle joint compared to the TL. The results of this study support our hypothesis. The demand on the whole body during the take-off phase was greater for the SDC jump, which showed greater peak vertical GRF and RFD when compared to the TL. The effect sizes of the differences in the jump conditions for both RFD and peak vertical GRF were large, indicating that the difference in whole-body demand between the two jumps was meaningful. Similarly, the mechanical demand specifically at the ankle joint was greater for the SDC when compared to the TL, as indicated by greater peak net joint moment and positive power. The effect sizes for the peak ankle moment and power were also very large, indicating meaningful differences in mechanical demand at the ankle joint between these two jumps. All variables showed identical trends, suggesting that the SDC elicits a greater mechanical demand when compared to the TL.

These net joint moment and power data imply that high demands were placed on the AT during jumping movements in ballet, specifically during the take-off phase. During the entire take-off phase for both jumps, the net ankle moments were plantarflexor (Figure 4) and, simultaneously, the net ankle powers were predominately positive (Figure 5). The concurrence of plantarflexor moment and positive power suggests that the plantarflexors would be acting concentrically. ${ }^{37}$ Because primary plantarflexors are gastrocnemius and soleus, the concentric action of these muscles would pull on and increase tension in the AT, thereby transferring the moment and power to the ground needed for the jump take-off. ${ }^{21}$ Based on the repetitive

Table I Kinetic Variables for the Take-off Phase of SDC and TL

\begin{tabular}{|l|l|l|l|l|l|}
\hline & Saut de chat & Temps levé & $t$-Value & $\boldsymbol{P}$ & Cohen's d \\
\hline Peak vertical force (N/kg) & $23.2 \pm 2.7$ & $21.2 \pm 2.3$ & $t_{14}=3.53$ & $0.003^{*}$ & 0.914 \\
Mean RFD (N/kg/s) & $103.3 \pm 35.6$ & $74.4 \pm 17.8$ & $t_{14}=3.83$ & $0.002^{*}$ & 0.987 \\
Flight time (s) & $0.35 \pm 0.04$ & $0.32 \pm 0.05$ & $t_{14}=3.37$ & $0.005^{*}$ & 0.869 \\
Peak ankle moment (Nm/kg) & $3.03 \pm 0.40$ & $2.61 \pm 0.38$ & $t_{13}=4.68$ & $<0.00 I^{*}$ & 1.238 \\
Peak ankle power (W/kg) & $20.7 \pm 4.7$ & $15.6 \pm 3.5$ & $t_{13}=8.53$ & $<0.00 I^{*}$ & 2.290 \\
\hline
\end{tabular}

Notes: *Denotes significant difference $(p<0.05)$. Data presented as mean \pm I standard deviation. 


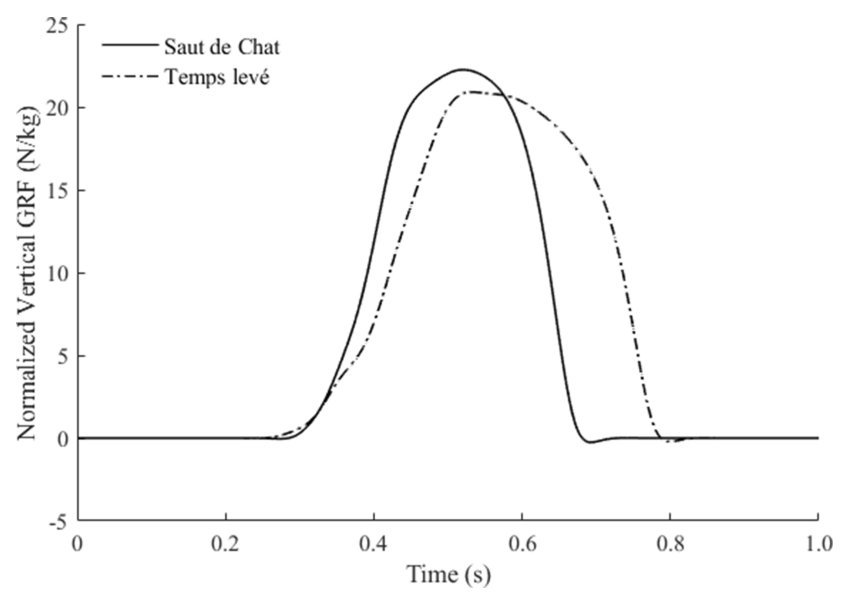

Figure 3 Representative normalized vertical GRF data during the take-off phase for both jumps.

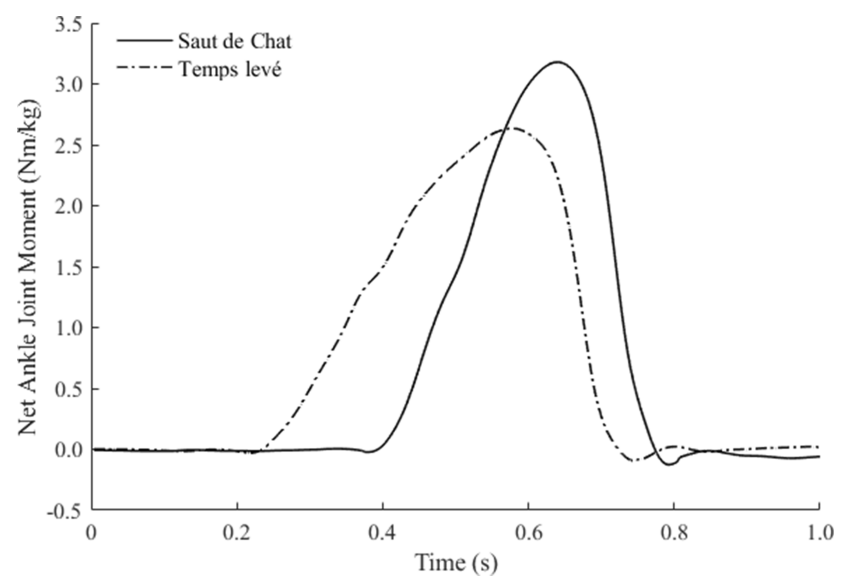

Figure 4 Representative net ankle joint moment during the take-off phase for both jumps. Positive moments are plantarflexor.

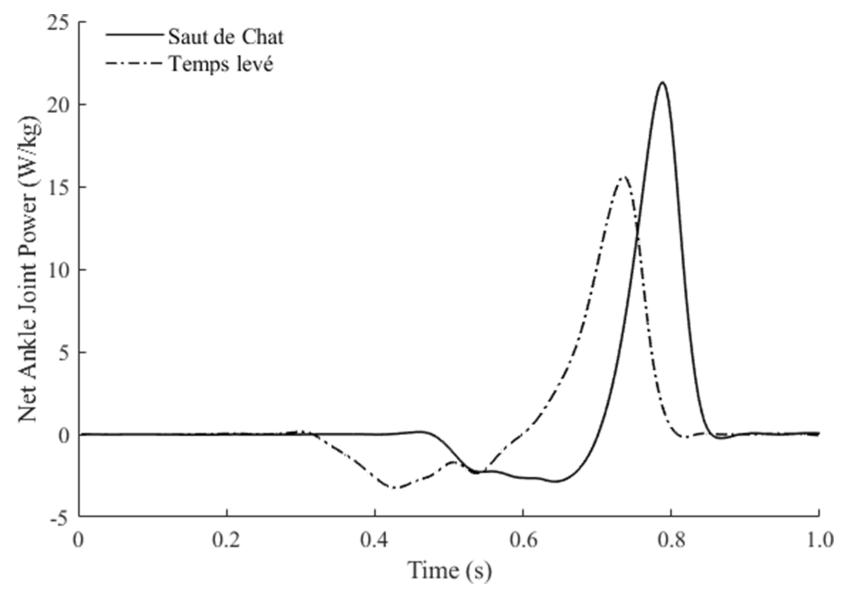

Figure 5 Representative net ankle joint power during the take-off phase for both jumps.

nature of dance movements, these strains may contribute to the high prevalence of the development of overuse injury at the ankle and Achilles tendinopathies associated with the take-off phase of these jumps. $5,7,8,12$

Net joint moment and power data reveal that the mechanical demands on the ankle plantarflexors and the AT are greater during the SDC than TL. During jumps, the plantarflexor moments accelerate the body upward and/or forward. ${ }^{38}$ These plantarflexor moments differ in magnitude depending on the direction of the jump. ${ }^{22}$ The SDC requires the movement of the body in both vertical and horizontal directions, ${ }^{3,17}$ whereas the TL is primarily a vertical jump. ${ }^{3}$ To fulfill the aesthetic requirement of the SDC (i.e., achieving splits while airborne), the vertical GRF and the flight time required of the SDC are greater compared to the TL (Table 1). Differences in demand between these two jumps may also be explained by how they are used in practice and performance. The SDC is trained as a more powerful jump compared to jumps such as the TL. ${ }^{3,17}$ Additionally, the aesthetic demand of each jump may temper a dancer's maximal effort by determining the optimal jump execution based on the timing of the musical cue or the intended effect of the jump. ${ }^{2}$ These considerations may also determine how often a jump is practiced and to what degree of effort. The high mechanical demand of the take-off phase of the jumps and repetitive nature of ballet training ${ }^{7,8}$ could increase the risk of overuse injury and Achilles tendinopathies. ${ }^{1,4,6}$ The risk of overuse injuries during ballet may be elucidated by determining the differences in mechanical demand at the ankle joint during the take-off phase of different jumps.

Since the SDC places a greater mechanical demand on the ankle joint than the TL, these two specific types of the jump could be used progressively in training. Such progression may be beneficial for both beginner and advanced ballet students. Intensive ballet training often begins at a young age, and high demand movements that are introduced early can impact the development of injuries. ${ }^{39,40}$ Even in advanced training, ensuring that lower demand jumps such as the TL are performed adequately before training, higher demand jumps such as the SDC may mitigate the development of overuse type injuries. ${ }^{41,42}$ This strategy could be applied at the beginning of the ballet season after they have taken time off to ensure high demand jumps are not performed when the dancer may be in a deconditioned state. ${ }^{43}$ Similarly, this type of progression could be used in rehabilitation for ballet dancers with AT rupture or AT tendinopathies. The mechanical demand on the ankle can be further reduced for jumps such as TL by limiting jump height until the correct form is observed, then increasing jump height of this jump, and 
then moving on to higher demand jumps such as the SDC. Such training progression may reduce the possibility of reinjury during rehabilitation by ensuring proper, progressive training of the AT.

Some limitations of the current study include the testing of ballet jumps in isolation, as well as the modification to the jump approach. In ballet training and performance, jumps are a part of a choreographed set of movements and are rarely performed as an isolated movement. Additionally, the SDC typically has a running approach ${ }^{17}$ compared to a single-step approach used in the current study. By controlling the approach strategy, we were able to control the confounding effects of the approach speed on the ground reaction forces and the ankle joint moment and power. However, by both isolating the jumps and not utilizing a running approach, the mechanical demand data collected in this study may be lower and not be indicative of true peak values achieved during ballet practice and performance. Another limitation of this study is that all jumps were tested with bare feet rather than in ballet shoes. We chose to test participants barefoot to control for possible variability in jump performance due to differences in shoe stiffness. ${ }^{4,13,39}$

Future studies should investigate the mechanical demand of additional types of ballet jumps so a comprehensive progression based on mechanical demand at the ankle joint can be determined and applied to training and rehabilitation programs. Additionally, the effects of upper extremity kinematics on ballet jumps, specifically during the take-off phase, should be observed. Upper extremity kinematics often determine the aesthetic quality of the movement in ballet. ${ }^{44}$ Therefore, upper extremity movement may play a large role in total take-off strategy and may alter the demand at the ankle for specific jumps depending on the desired aesthetic.

Our data show that the mechanical demand at the ankle joint is significantly greater for the SDC than the TL. The results of the current study could be used to begin a compendium of ballet jumps organized by mechanical demand at the ankle. These findings have implications for injury prevention as well as potential for developing effective training and rehabilitation progressions for the classically trained ballet dancer.

\section{Abbreviations}

SDC, saut de chat; TL, temps levé; AT, Achilles tendon; GRF, ground reaction force; RFD, rate of force development.

\section{Ethics Approval and Informed Consent}

All participants signed a written informed consent prior to participation. The Western Washington University Institutional Review Board approved the study.

\section{Disclosure}

The authors report no conflicts of interest in this work.

\section{References}

1. Koutedakis Y, Jamurtas A. The dancer as a performing athlete: physiological considerations. Sports Med. 2004;34(10):651-661. doi:10.2165/00007256-200434100-00003

2. Schantz PG, Astrand PO. Physiological characteristics of classical ballet. Med Sci Sports Exerc. 1984;16(5):472-476. doi:10.1249/00005768-198 410000-00009

3. Triegaart E. Congress: the needs of the dancer. South Afr J Sports Med. 1995;2(2):16-18.

4. Schon LC, Weinfeld SB. Lower extremity musculoskeletal problems in dancers. Curr Opin Rheumatol. 1996;8(2):130-142. doi:10.1097/ 00002281-199603000-00008

5. Kannus P. Etiology and pathophysiology of chronic tendon disorders in sports. Scand J Med Sci Sports. 1997;7(2):78-85. doi:10.1111/ j.1600-0838.1997.tb00123.x

6. Fernandez-Palazzi F, Rivas S, Mujica P. Achilles tendinitis in ballet dancers. Clin Orthop Relat Res. 1990;(257):257-261.

7. Sobrino FJ, Guillen P. Overuse injuries in professional ballet: influence of age and years of professional practice. Orthop J Sports Med. 2017;5(6):2325967117712704. doi:10.1177/2325967117712704

8. Nilsson C, Leanderson J, Wykman A, Strender LE. The injury panorama in a Swedish professional ballet company. Knee Surg Sports Traumatol Arthrosc. 2001;9(4):242-246. doi:10.1007/s001670100 195

9. O’Loughlin PF, Hodgkins CW, Kennedy JG. Ankle sprains and instability in dancers. Clin Sports Med. 2008;27(2):247-262. doi:10.1016/j.csm.2007.12.006

10. Kadel NJ. Foot and ankle injuries in dance. Phys Med Rehabil Clin N Am. 2006;17(4):813-826. doi:10.1016/j.pmr.2006.06.006

11. Egger AC, Berkowitz MJ. Achilles tendon injuries. Curr Rev Musculoskelet Med. 2017;10(1):72-80. doi:10.1007/s12178-0179386-7

12. Kulig K, Loudon JK, Popovich JM Jr., Pollard CD, Winder BR. Dancers with achilles tendinopathy demonstrate altered lower extremity takeoff kinematics. J Orthop Sports Phys Ther. 2011;41 (8):606-613. doi:10.2519/jospt.2011.3580

13. Chockley C. Ground reaction force comparison between jumps landing on the full foot and jumps landing en pointe in ballet dancers. J Dance Med Sci. 2008;12(1):5-8.

14. Lee HH, Lin CW, Wu HW, Wu TC, Lin CF. Changes in biomechanics and muscle activation in injured ballet dancers during a jump-land task with turnout (Sissonne Fermee). J Sports Sci. 2012;30(7):689-697. doi:10.1080/02640414.2012.663097

15. Simpson KJ, Kanter L. Jump distance of dance landings influencing internal joint forces: I. Axial forces. Med Sci Sports Exerc. 1997;29 (7):916-927. doi:10.1097/00005768-199707000-00011

16. Simpson KJ, Pettit M. Jump distance of dance landings influencing internal joint forces: II. Shear forces. Med Sci Sports Exerc. 1997;29 (7):928-936. doi:10.1097/00005768-199707000-00012

17. Jarvis DN, Kulig K. Lower extremity biomechanical demands during saut de chat leaps. Med Probl Perform Art. 2016;31(4):211-217. doi:10.21091/mppa.2016.0000 
18. Maffiuletti NA, Aagaard P, Blazevich AJ, Folland J, Tillin N, Duchateau J. Rate of force development: physiological and methodological considerations. Eur J Appl Physiol. 2016;116 (6):1091-1116. doi:10.1007/s00421-016-3346-6

19. Suzuki Y, Kobayashi Y, Takizawa M. Effects of joint moments on horizontal and vertical velocities of body mass center during jumping in different directions. Int J Sport Health Sci. 2018;16.

20. Wang LI, Peng HT. Biomechanical comparisons of single- and double-legged drop jumps with changes in drop height. Int $J$ Sports Med. 2014;35(6):522-527. doi:10.1055/s-0033-1345133

21. Farris DJ, Lichtwark GA, Brown NA, Cresswell AG. The role of human ankle plantar flexor muscle-tendon interaction and architecture in maximal vertical jumping examined in vivo. $J$ Exp Biol. 2016;219(Pt 4):528-534. doi:10.1242/jeb.126854

22. Moo EK, Peterson DR, Leonard TR, Kaya M, Herzog W. In vivo muscle force and muscle power during near-maximal frog jumps. PLoS One. 2017;12(3):e0173415. doi:10.1371/journal.pone.0173415

23. Fukashiro S, Besier TF, Barrett R, Cochrane J, Nagano A, Lloyd DG Direction control in standing horizontal and vertical jumps. Int J Sport Health Sci. 2005;3.

24. Wylon M, Allen N, Angioi M, Nevill A, Twitchett E. Anthropometric factors affecting vertical jump height in ballet dancers. J Dance Med Sci. 2005;10(3):106-110.

25. Strom AC, Casillas MM. Achilles tendon rehabilitation. Foot Ankle Clin. 2009;14(4):773-782. doi:10.1016/j.fcl.2009.08.003

26. Schmitt H, Kuni B, Sabo D. Influence of professional dance training on peak torque and proprioception at the ankle. Clin J Sport Med. 2005;15(5):331-339. doi:10.1097/01.jsm.0000181437.41268.56

27. Morrin N, Redding E. Acute effects of warm-up stretch protocols on balance, vertical jump height and range of motion in dancers. $J$ Dance Med Sci. 2013;17(1):34 40. doi:10.12678/1089-313X.17.1.34

28. Imura A, Iino Y. Comparison of lower limb kinetics during vertical jumps in turnout and neutral foot positions by classical ballet dancers. Sports Biomech. 2017;16(1):87-101. doi:10.1080/14763141.2016. 1205122

29. Carter SL, Duncan R, Weidemann AL, Hopper LS. Lower leg and foot contributions to turnout in female pre-professional dancers: a 3D kinematic analysis. J Sports Sci. 2018;36(19):2217-2225. doi:10.1080/ 02640414.2018 .1446386

30. Jensen RL, Ebben WP. Quantifying plyometric intensity via rate of force development, knee joint, and ground reaction forces. J Strength Cond Res. 2007;21(3):763-767. doi:10.1519/R-18735.1

31. Bailey CA, Sato K, Burnett A, Stone MH. Force-production asymmetry in male and female athletes of differing strength levels. Int J Sports Physiol Perform. 2015;10(4):504-508. doi:10.1123/ ijspp.2014-0379
32. Harry JR, Barker LA, Eggleston JD, Dufek JS. Evaluating performance during maximum effort vertical jump landings. $J \mathrm{Appl}$ Biomech. 2018;34(5):403-409. doi:10.1123/jab.2017-0172

33. Mcmahon JJ, Jones PA, Comfort P. A correction equation for jump height measured using the just jump system. Int J Sports Physiol Perform. 2016;11(4):555-557. doi:10.1123/ijspp.2015-0194

34. Plug-in Gait Reference Guide 2017. Available from: https://docs. vicon.com/display/Nexus 26/Plug-in\%20Gait\%20Reference\% 20Guide. Accessed November 22, 2019.

35. Dempster WT. Space Requirements of the Seated Operator, Geometrical, Kinematic, and Mechanical Aspects of the Body with Special Reference to the Limbs. East Lansing: Michigan State University; 1955.

36. Cohen J. Statistical Power Analysis for the Behavioral Sciences. 2nd ed. Hillsdale, NJ: Lawrence Erlbaum Associates; 1988.

37. Winter DA. Biomechanics and Motor Control of Human Movement. 4th ed. Hoboken, NJ: John Wiley \& Sons; 2009.

38. Nejadfard A, Schutz S, Mianowski K, Vonwirth P, Berns K Coordination of the biarticular actuators based on mechanical output power in an explosive jump experiment. 2018 IEEE/ASME International Conference on Advanced Intelligent Mechatronics (AIM); 2018:220-225; Auckland, New Zealand.

39. Weiss DS, Rist RA, Grossman G. When can I start pointe work? Guidelines for initiating pointe training. J Dance Med Sci. 2009;13 (1):90-92

40. Hamilton D, Aronsen P, Løken JH, et al. Dance training intensity at 11-14 years is associated with femoral torsion in classical ballet dancers. Br J Sports Med. 2006;40(4):299-303. doi:10.1136/ bjsm.2005.020941

41. Bayliss AJ, Weatherholt AM, Crandall TT, et al. Achilles tendon material properties are greater in the jump leg of jumping athletes. J Musculoskelet Neuronal Interact. 2016;16(2):105-112.

42. Smith PJ, Gerrie BJ, Varner KE, McCulloch PC, Lintner DM, Harris JD. Incidence and prevalence of musculoskeletal injury in ballet: a systematic review. Orthop J Sports Med. 2015;3 (7):2325967115592621. doi:10.1177/2325967115592621

43. Koutedakis Y, Myszkewycz L, Soulas D, Papapostolou V, Sullivan I, Sharp NC. The effects of rest and subsequent training on selected physiological parameters in professional female classical dancers. Int J Sports Med. 1999;20(6):379-383. doi:10.1055/s-2007-971148

44. Kalichova M. Biomechanical analysis of the basic classical dance jump - the grand jete. Int J Sport Health Sci. 2011;5(11):1363-1367. doi:10.1177/193229681100500607
Open Access Journal of Sports Medicine is an international, peerreviewed, open access journal publishing original research, reports, reviews and commentaries on all areas of sports medicine. The manuscript management system is completely online and includes a very quick and fair peer-review system. Visit http://www.dovepress. com/testimonials.php to read real quotes from published authors. 